\title{
BAY 87-2243, a novel inhibitor of hypoxia-induced gene activation, improves local tumor control after fractionated irradiation in a schedule-dependent manner in head and neck human xenografts
}

Linda Helbig 1,2, Lydia Koi ${ }^{1,2,3}$, Kerstin Brüchner ${ }^{2,4}$, Kristin Gurtner ${ }^{2}$, Holger Hess-Stumpp ${ }^{5}$, Kerstin Unterschemmann ${ }^{5}$, Michael Baumann ${ }^{1,2,3,4}$, Daniel Zips ${ }^{6,7 \dagger}$ and Ala Yaromina $a^{1,2,8^{*}+}$

\begin{abstract}
Background: The transcription factor hypoxia-inducible factor-1 (HIF-1) pathway plays an important role in tumor response to cytotoxic treatments. We investigated the effects of a novel small molecule inhibitor of mitochondrial complex I and hypoxia-induced HIF-1 activity BAY-87-2243, on tumor microenvironment and response of human squamous cell carcinoma (hSCC) to clinically relevant fractionated radiotherapy (RT) with and without concomitant chemotherapy.

Methods: When UT-SCC-5 hSCC xenografts in nude mice reached $6 \mathrm{~mm}$ in diameter BAY-87-2243 or carrier was administered before and/or during RT or radiochemotherapy with concomitant cisplatin (RCT). Local tumor control was evaluated 150 days after irradiation and the doses to control $50 \%$ of tumors $\left(\mathrm{TCD}_{50}\right)$ were compared between treatment arms. Tumors were excised at different time points during BAY-87-2243 or carrier treatment for western blot and immunohistological investigations.

Results: BAY-87-2243 markedly decreased nuclear HIF-1a expression and pimonidazole hypoxic fraction already after 3 days of drug treatment. BAY-87-2243 prior to RT significantly reduced TCD ${ }_{50}$ from 123 to 100 Gy ( $p=0.037$ ). Additional BAY-87-2243 application during RT did not decrease $T_{C D}$. BAY-87-2243 before and during radiochemotherapy did not improve local tumor control.

Conclusions: Pronounced reduction of tumor hypoxia by application of BAY-87-2243 prior to RT improved local tumor control. The results demonstrate that radiosensitizing effect importantly depends on treatment schedule. The data support further investigations of HIF-1 pathway inhibitors for radiotherapy and of predictive tests to select patients who will benefit from this combined treatment.
\end{abstract}

Keywords: HIF pathway inhibition, Cisplatin, Fractionated radiation, Local tumor control, Tumor microenvironment, Human tumor xenograft

\footnotetext{
* Correspondence: ala.yaromina@maastrichtuniversity.nl

${ }^{\dagger}$ Equal contributors

'OncoRay - National Center for Radiation Research in Oncology, Medical

Faculty Carl Gustav Carus, TU Dresden, Dresden, Germany

${ }^{2}$ Department of Radiation Oncology, University Hospital Carl Gustav Carus,

TU Dresden, Dresden, Germany

${ }^{8}$ Department of Radiation Oncology (MAASTRO Lab), GROW - School for

Oncology and Developmental Biology, Maastricht University Medical Centre,

Maastricht, The Netherlands

Full list of author information is available at the end of the article
}

\section{Biomed Central}

(c) 2014 Helbig et al.; licensee BioMed Central Ltd. This is an Open Access article distributed under the terms of the Creative Commons Attribution License (http://creativecommons.org/licenses/by/4.0), which permits unrestricted use, distribution, and reproduction in any medium, provided the original work is properly credited. The Creative Commons Public Domain Dedication waiver (http://creativecommons.org/publicdomain/zero/1.0/) applies to the data made available in this article, unless otherwise stated. 


\section{Introduction}

Many solid tumors express hypoxia-inducible factor- $1 \alpha$ $(\mathrm{HIF}-1 \alpha)$, which is associated with poor prognosis after surgery, radiotherapy, and chemotherapy in several cancer types [1-5]. Tumor hypoxia, among other stress conditions [6-8], is recognized as a major regulator of multiple HIF-1-mediated pathways which promote cell survival [9]. Hypoxia leads to the stabilization and accumulation of HIF- $1 \alpha$ protein, which translocates to the nucleus and forms a heterodimer with its partner HIF-1 $\beta$. This transcriptional complex induces the transcription of numerous genes with adaptive functions, e.g. vascular endothelial growth factor and glucose transporter 1 to increase oxygen availability and to allow metabolic adaptation to oxygen deprivation.

Pharmacological or genetic targeting of HIF-1 sensitized tumor cells to radiation and chemotherapeutic DNA damaging agents and decreased tumor growth [10-15]. Beside direct radiosensitization of tumor cells caused by HIF-1 inhibition other mechanisms such as radiosensitization of tumor vasculature or reduction of tumor hypoxia have been shown to contribute to the enhanced effect of radiation therapy [16-19]. Resistance of hypoxic tumor cells to chemotherapy was attributed to several factors including poor drug distribution, reduced drug uptake, activation of genes leading to a drug-resistant phenotype [20]. Recent studies have demonstrated an important role of HIF-1 in resistance to chemotherapeutic agents such as platinum-containing anti-cancer drugs, e.g. through regulation of XPA (xeroderma pigmentosum group A) protein that senses DNA damage and recruits other DNA repair proteins to the damaged template in the nucleotide excision repair pathway $[21,22]$.

BAY-87-2243 inhibits mitochondrial production of reactive oxygen species (ROS) by blocking mitochondrial complex I, which subsequently reduces hypoxia-induced HIF-1 activity [23]. Being encouraged by our recent findings using the compound BAY-84-7296 with the same mode of action but lower on-target efficiency as its derivative BAY-87-2243, which completely resolved tumor hypoxia and pronouncedly increased local tumor control after irradiation with large single doses in two different hSCCs of head and neck, UT-SCC-14 and UT-SCC-5, in vivo [24], we tested in the present study whether BAY-87-2243 leads to the reduction of tumor hypoxia and improves the outcome of clinically relevant fractionated irradiation with and without concomitant cisplatin treatment. The fractionation protocol with 30 fractions over 6 weeks was chosen to account for potential interactions between the compound and radiobiological mechanisms of fractionated irradiation such as repopulation, reoxygenation, recovery and redistribution, which by design did not contribute to local tumor control after single dose irradiation. UT-SCC-5 hSCC was chosen for the experiments because this tumor model is more radioresistant and exhibits higher expression of HIF- $1 \alpha$ and hypoxic fraction as compared with UT-SCC-14 [24,25]. The efficacy of various combination regimens have been tested using a series of $\mathrm{TCD}_{50}$ (dose to cure $50 \%$ of tumors) assays in nude mice. We show that radiosensitizing effect of BAY-87-2243 with fractionated irradiation depends on treatment schedule, which may provide important information for the design of clinical trials.

\section{Methods}

\section{BAY-87-2243, cisplatin}

BAY-87-2243 was developed by Bayer Pharma AG. For the experiments BAY-87-2243 was dissolved in carrier solution (10\% ethanol, $40 \%$ Solutol $^{\circ}$ HS15, 50\% sterile distilled water) and administered orally by gavage $(9 \mathrm{mg} / \mathrm{kg} /$ body weight $[$ b.w.]). Control animals were treated with the carrier solution. Cisplatin (Calbiochem, Germany, $3 \mathrm{mg} /$ $\mathrm{kg} / \mathrm{b} . \mathrm{w}$.$) dissolved in saline (\mathrm{NaCl} 0.9 \%)$ was administered intraperitoneally (i.p.).

\section{Animals and tumor model}

The experiments were performed using 7 to 14 week-old male and female NMRI (nu/nu) nude mice obtained from the pathogen-free animal breeding facility (Experimental Centre, Medical Faculty, TU Dresden, Germany). Two to five days prior to tumor transplantation the nude mice received a total body irradiation (4 Gy, $200 \mathrm{kV}$ X-rays, $0.5 \mathrm{~mm} \mathrm{Cu}$-filter, $\sim 1 \mathrm{~Gy} / \mathrm{min}$ ) for further immunosuppression. The experiments and the animal facilities were approved according to the institutional guidelines and the German animal welfare regulations.

The experiments were performed using the established hSCC of the tongue UT-SCC- 5 with hypoxia-induced activation of HIF-1 $\alpha$ [26]. Small pieces of a source tumor were transplanted subcutaneously into the right hind-leg of anesthetized mice $(120 \mathrm{mg} / \mathrm{kg}$ b.w. ketamine i.p. and $16 \mathrm{mg} / \mathrm{kg}$ xylazine i.p.). The volume doubling time, histological examinations and DNA-microsatellite profile confirmed the identity of the transplanted UT-SCC-5 xenografts.

\section{Tumor sampling}

BAY-87-2243 or carrier solution were applied once tumors reached $4 \mathrm{~mm}\left(\sim 33 \mathrm{~mm}^{3}\right)$ in diameter until the tumors reached $7 \mathrm{~mm}\left(\sim 180 \mathrm{~mm}^{3}\right)$ or once tumors reached $6 \mathrm{~mm}$ for 3, 5, or 7 days. At these time points the tumors were excised $24 \mathrm{~h}$ after the last carrier or BAY-87-2243 administration (3-9 mice per treatment group) for immunohistochemistry or western blotting. Prior to tumor harvesting the hypoxia marker pimonidazole (Natural Pharmacia International, Inc., Research Triangle Park, NC, USA; $0.1 \mathrm{mg} / \mathrm{g}$ b.w., dissolved at $10 \mathrm{mg} / \mathrm{ml}$ in $0.9 \% \mathrm{NaCl}$, i.p.) was injected one hour 
before excision as well as the perfusion marker Hoechst 33342 (Sigma Aldrich, Deisenhofen, Germany; 0.75 mg in PBS, intravenously [i.v.]) one minute before excision. Half of the tumor was immediately snap frozen in liquid nitrogen and stored at $-80^{\circ} \mathrm{C}$. The second half was fixed overnight in $4 \%$ formalin and embedded in paraffin.

\section{Tumor growth delay and $\mathrm{TCD}_{50}$ assay}

In tumor growth delay assay randomized mice were treated daily with carrier $(n=39)$ or BAY-87-2243 $(n=41)$ when tumors reached a size of $4 \mathrm{~mm}$ in diameter until the tumors grew to $7 \mathrm{~mm}$ in diameter. Time to reach $7 \mathrm{~mm}$ was compared between treatment groups.

In $\mathrm{TCD}_{50}$ assay six experimental treatments were tested combining fractionated radiotherapy (RT) or cisplatin based radiochemotherapy (RCT) with BAY-87-2243:

1) RT: 30 fractions delivered over 6 weeks. Carrier was applied before and during radiotherapy.

2) BAY-87-2243 + RT: pre-treatment with BAY-87-2243 followed by RT with carrier.

3) BAY-87-2243 + RT/BAY-87-2243: treatment with BAY-87-2243 before and during RT.

4) RCT: 30 fractions over 6 weeks combined with cisplatin once per week after irradiation. Carrier was applied before and during RCT.

5) RCT/BAY-87-2243: pre-treatment with carrier followed by RCT with BAY-87-2243.

6) BAY-87-2243 + RCT/BAY-87-2243: treatment with BAY-87-2243 before and during RCT.

Pre-treatment with carrier or BAY-87-2243 was always performed on three consecutive days followed by RT 24 $\mathrm{h}$ after the last application. Carrier or BAY-87-2243 was administered after each radiation fraction during radiotherapy.

Treatments started when tumor size reached $6 \mathrm{~mm}$ in diameter. Animals were randomly allocated in six groups with total doses between 60 Gy and 160 Gy (5-12 mice per dose group) delivered in 30 equal fractions (dose per fraction between 2 and 5.3 Gy). All irradiations were performed under normal blood flow conditions without anaesthesia. During irradiation animals were immobilized using plastic tubes fixed on a lucite plate and the tumor-bearing leg was positioned in the irradiation field by a foot holder distal to the tumor. The tumor diameters were measured using a caliper twice per week and once per week 90 days after irradiation. Tumor volume was calculated by $\pi / 6 \cdot a \cdot b^{2}$, where a is the longest and $\mathrm{b}$ is the perpendicular shorter tumor diameter. When tumor volume increased for three consecutive measurements after shrinkage or when tumors continued to grow without shrinkage they were scored as recurrence. The animals were sacrificed when the recurrent tumor reached the diameter of $15 \mathrm{~mm}$ or when the animal appeared to suffer. A total of 343 mice were observed after treatment for a maximum time of 150 days, which is sufficient to detect all local failures in UT-SCC-5 [26]. Ninety five percent of 200 local failure events occurred before day 69 and the latest recurrence was scored at day 150. A total of 71 mice censored between 21 and 149 days were included in the analysis [25]. Fitting of the local control data, calculation of the $\mathrm{TCD}_{50}$ values, of $95 \%$ confidence intervals for $\mathrm{TCD}_{50}$ and of $\mathrm{p}$-values for comparisons of treatment groups were performed as described previously [25]. Enhancement ratio (ER) was calculated as the ratio between the $\mathrm{TCD}_{50}$ value of the control group and the $\mathrm{TCD}_{50}$ of the experimental group.

\section{Western blot analysis of HIF-1a}

Western blotting was performed one time according to the established protocol as described previously [24]. Protein samples were prepared using the NE-PER Nuclear and Cytoplasmic KIT (Thermo Scientific, Germany) according to the manufacturer's instructions. Antibodies used were mouse monoclonal anti-human HIF-1 $\alpha$ (1:250, BD Biosciences, USA) and rabbit polyclonal anti-histoneH2B (1:500, Imgenex, USA) and anti-Calpain 1 (1:1000, Cell Signaling, USA) served as the loading controls for nuclear or cytoplasmic cell compartments, respectively. Nuclear HIF-1 $\alpha$ band intensities were normalized to histone-H2B levels.

\section{Histological studies}

Two $10 \mu \mathrm{m}$ frozen cross-sections from tumor centre were stained for pimonidazole (rabbit anti-pimonidazole antisera, Burlington, USA) and CD31 (rat anti-mouse CD31, clone MEC 13.3, PharMingen/BD Biosciences, Heidelberg, Germany), scanned and blindly analysed as described in detail previously [26]. After scanning, the same tumor sections were stained with haematoxylin and eosin for identification of viable and necrotic tumour subareas by morphological criteria. The pimonidazole hypoxic fraction (pHF) and the relative vascular area (RVA) were calculated as the percentage of the viable tumor area stained for pimonidazole or CD31, respectively. The fraction of perfused vessels (PF) was calculated as the percentage of the vascular area overlapped with Hoechst 33342 perfusion marker. Necrotic fraction (NF) was determined as the necrotic tumor area divided by the total tumor area. The immunohistochemistry staining protocol for HIF-1 $\alpha$ (mouse monoclonal anti-human HIF-1 $\alpha$, BD Biosciences, USA) has previously been described in detail $[24,27]$.

\section{Statistical analysis}

Normality of distributions was tested using the skewness and kurtosis test. Mean values were compared using the 
independent sample t-test. Non-parametric Mann-Whitney test was used to compare non-normal distributions. P-values were adjusted for multiple comparisons using the Scheffe correction when relevant. Statistical analysis was performed using a commercial software package STATA/SE 8.0 (STATA Corporation, College Station, TX, USA). P-values less or equal to 0.05 were considered as statistically significant.

\section{Results}

To test the inhibitory effect of BAY-87-2243 on HIF- $1 \alpha$ protein expression, tumors were treated with the drug for 3,5 or 7 consecutive days. Nuclear HIF- $1 \alpha$ protein levels were strongly suppressed after 3 days of drug treatment (Figure 1). Cytoplasmic cell extracts showed none or very weak HIF- $1 \alpha$ protein expression (data not shown). To study the kinetics of changes in tumor microenvironment induced by BAY-87-2243, hypoxia, vasculature and perfused vessels were examined in tumors after 3, 5 and 7 days of drug treatment. Three daily applications of BAY-87-2243 markedly reduced pHF to $1 \%$ as compared with $25 \%$ in carrier-treated tumors $(\mathrm{p}<$
0.0001 , Figures 2 and 3a). This decrease in pHF remained after BAY-87-2243 treatment for 5 and 7 days. A statistically significant reduction in RVA was found 5 and 7 days after BAY-87-2243 treatment as compared with carrier-treated tumors $(\mathrm{p}=0.033$ and $\mathrm{p}=0.026$, respectively, Figure 3a). BAY-87-2243 did not affect PF at any time point. Necrotic fraction was statistically significantly lower only after 3 days of drug treatment $(\mathrm{p}=0.018)$.

Anti-tumor effects of BAY-87-2243 were tested using daily application from $4 \mathrm{~mm}$ tumor diameter to $7 \mathrm{~mm}$. BAY-87-2243 alone significantly inhibited the growth of UT-SCC-5 tumors. The median time to reach $7 \mathrm{~mm}$ tumor diameter was 18 days for BAY-87-2243 as compared with 11 days for carrier treated tumors $(\mathrm{p}<0.0001$, Figure 3b). Administration of BAY-87-2243 for about 18 days significantly reduced HIF- $1 \alpha$ protein expression (data not shown) as well as pHF (mean 2.4\% (BAY-872243 ) vs. $17.6 \%$ (carrier), $\mathrm{p}<0.0001$ ), and NF (mean 9\% vs. $35.6 \%, \mathrm{p}=0.0002)$, whereas RVA and PF remained unchanged.

To test whether BAY-87-2243 improves local tumor control after clinically relevant fractionated irradiation

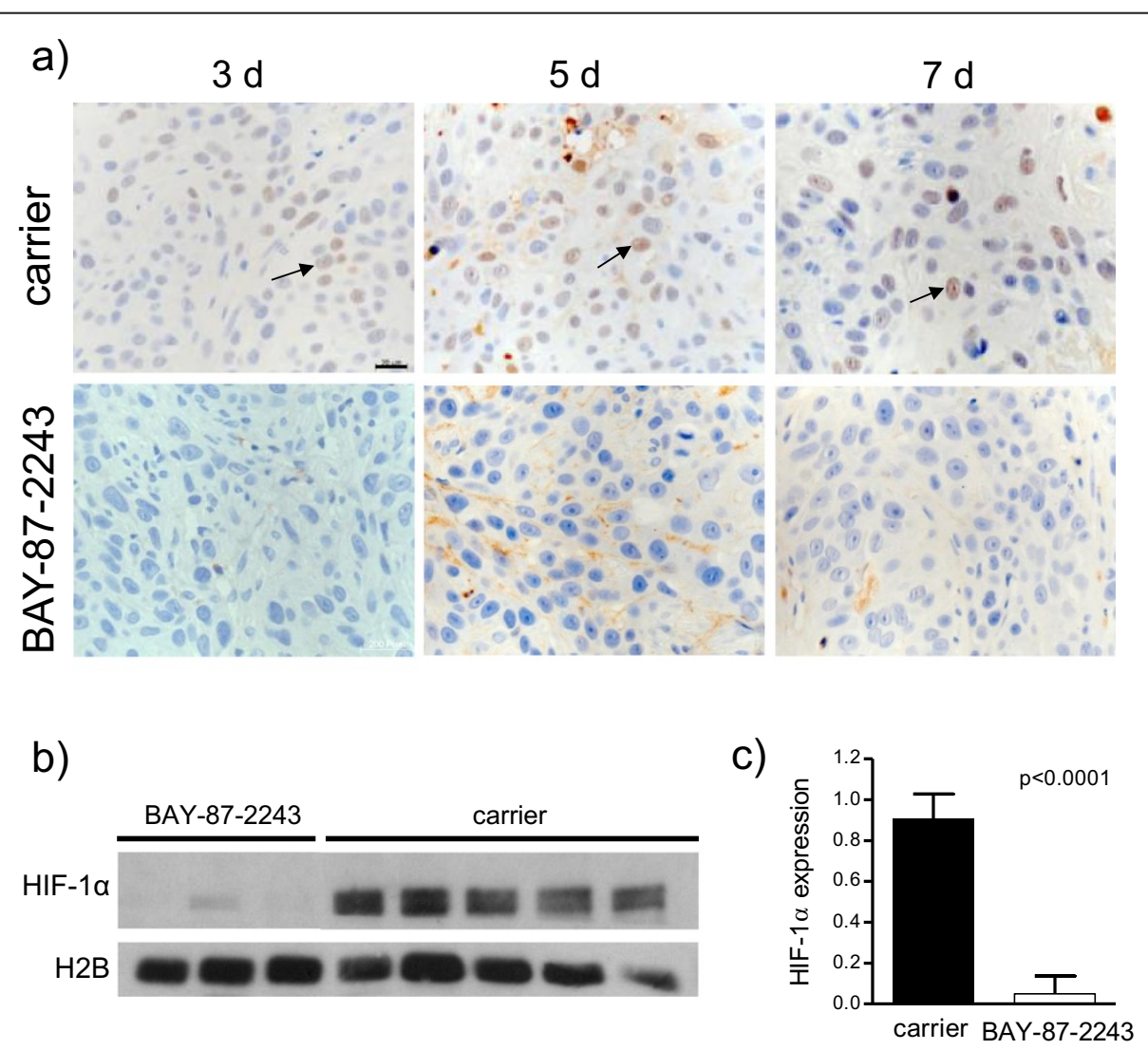

Figure 1 BAY-87-2243 inhibits nuclear HIF-1a protein expression. UT-SCC-5 tumors were treated either with BAY-87-2243 or carrier for 3, 5, or 7 days and were excised $24 \mathrm{~h}$ after end of treatment: a) immunohistochemistry, black arrows indicate the positive nuclear HIF-1a staining, scale bar: $20 \mu \mathrm{m}$; b) western blots of nuclear HIF-1a from different animals 3 days after BAY-87-2243 application; c) quantification of western blots by densitometry relative to histone-H2B, means $\pm \mathrm{SD}$. 


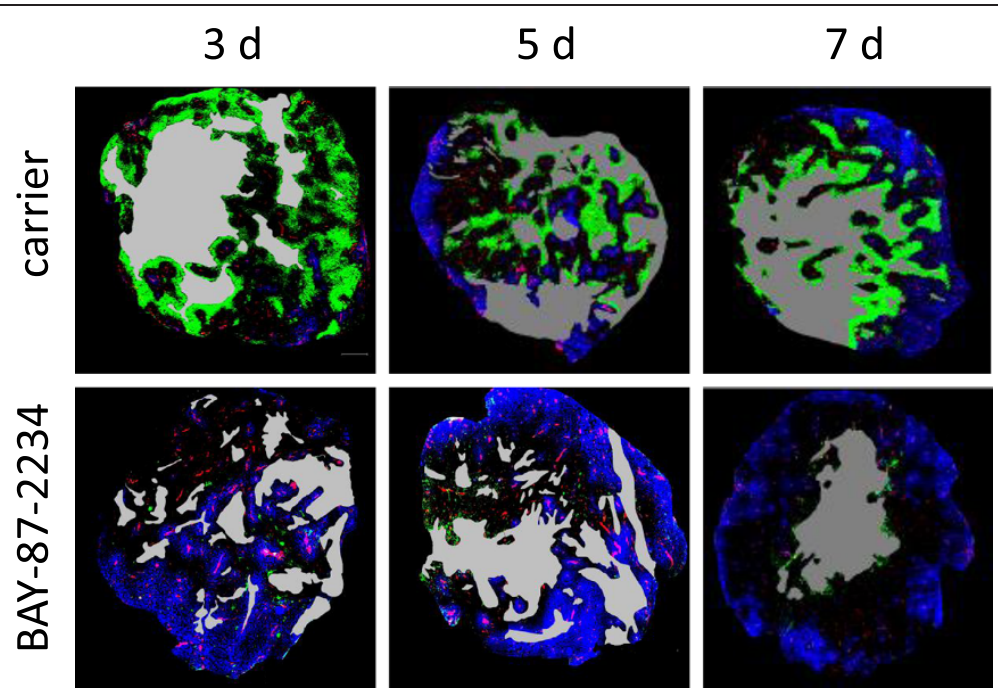

Figure 2 Pseudo-coloured images of cross-sections from tumors excised $24 \mathrm{~h}$ after end of treatment with BAY-87-2243 or carrier for 3, 5 or 7 days. Green: hypoxia, pimonidazole; blue: perfusion, Hoechst 33342; red: vascular endothelium, CD31; grey: necrotic areas. Scale bar: 500 m.
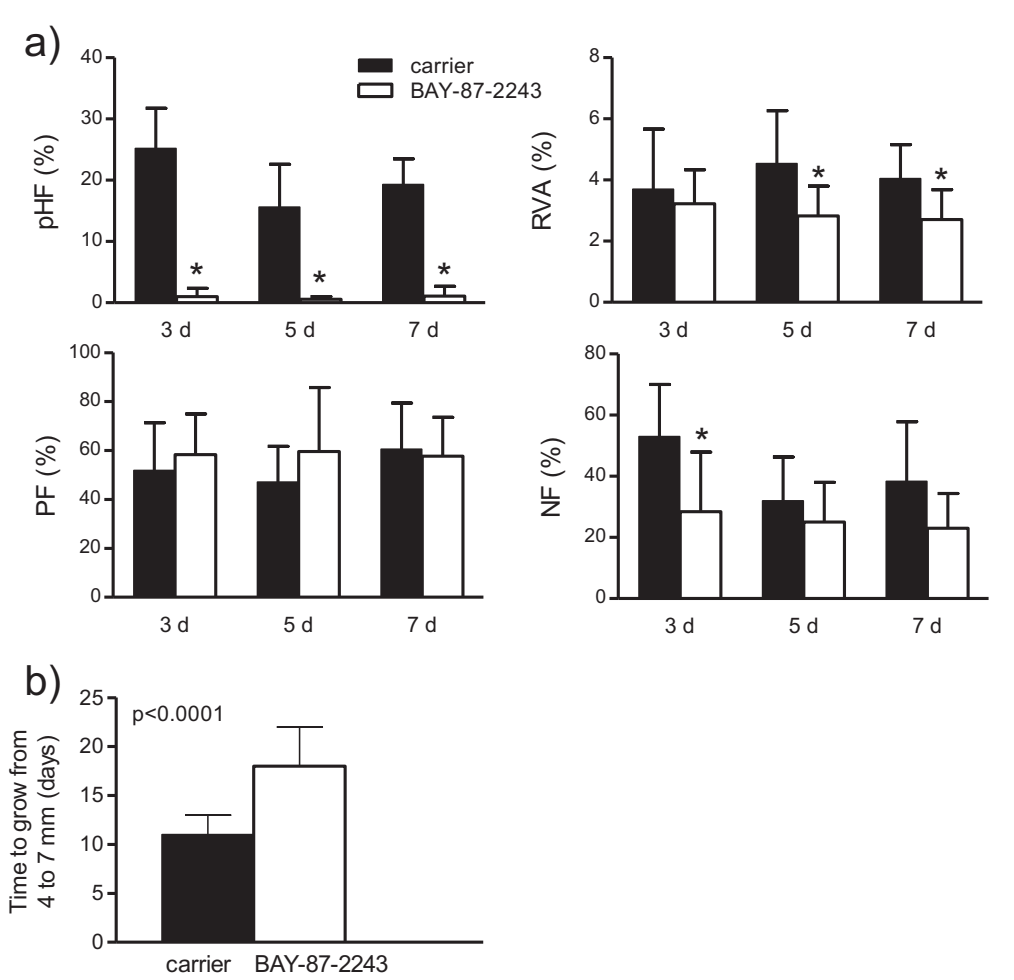

Figure 3 Effects of BAY-87-2243 on tumor microenvironment and tumor growth. a) Pimonidazole hypoxic fraction (pHF), relative vascular area (RVA), fraction of perfused vessels (PF), and necrotic fraction (NF) in UT-SCC-5 tumors $24 \mathrm{~h}$ after end of treatment with BAY-87-2243 or carrier for 3, 5, or 7 days ( $n=7-9$ tumors per time point and treatment group). b) Time to grow from 4 to $7 \mathrm{~mm}$ tumor diameter. UT-SCC-5 tumors were treated daily with BAY-87-2243 or carrier. Bars represent means \pm SD of histological parameters or medians \pm MAD (median absolute deviation) of growth times. Asterisks indicate significant differences from carrier control. 
(RT), the administration of BAY-87-2243 for 3 days was either halted before irradiation with 30 fractions within 6 weeks or was continued during irradiation. Whereas pre-treatment with BAY-87-2243 for 3 days prior to irradiation (BAY-87-2243+RT) significantly reduced $\mathrm{TCD}_{50}$, the decrease in $\mathrm{TCD}_{50}$ after concomitant BAY87-2243 treatment during irradiation (BAY-87-2243 + RT/BAY-87-2243) was less pronounced and statistically not significant (Table 1, Figure 4a). The enhancement ratio greater than 1 indicates that the dose to achieve the same local control rate (i.e. $\mathrm{TCD}_{50}$ ) is reduced by BAY-87-2243 in comparison with radiation alone. To test whether BAY-87-2243 decreases $\mathrm{TCD}_{50}$ after fractionated radiochemotherapy (RCT) BAY-87-2243 was combined with cisplatin and irradiation with 30 fractions within 6 weeks. The $\mathrm{TCD}_{50}$ after RCT was lower by a factor of 1.23 in comparison with RT alone. This effect was only marginally significant $(\mathrm{p}=0.09)$ likely due to considerable intertumoral heterogeneity in response to RCT as suggested by shallow dose-response curve (Figure 4b). Local tumor control was not significantly improved if BAY-87-2243 was administered during fractionated irradiation combined with weekly administration of cisplatin (RCT/BAY-87-2243). Pre-treatment with BAY-87-2243 for 3 days in addition to continuous BAY-87-2243 application during RCT (BAY-87-2243 + RCT/BAY-87-2243) also did not result in reduction of $\mathrm{TCD}_{50}$ (Table 1, Figure 4b).

\section{Discussion}

To our knowledge this experimental study demonstrates for the first time that BAY-87-2243, an inhibitor of hypoxia-induced gene activation, improves local tumor control after clinically relevant fractionated irradiation. The novelty of our findings is given by the fact that here

Table 1 Summary of $\mathrm{TCD}_{50}$ values and enhancement ratios (ER) for different treatments

\begin{tabular}{llc}
\hline Experimental arm & $\begin{array}{l}\text { TCD }_{\mathbf{5 0}}[\mathbf{9 5 \%} \text { Cl] (Gy) } \\
\text { (p-value vs. control) }\end{array}$ & ER \\
\hline 1) $\mathrm{RT}$ & $122.7[108 ; 149]$ & - \\
& - & \\
2) $\mathrm{BAY}-87-2243+\mathrm{RT}$ & $99.7[88 ; 117]$ & 1.23 \\
& $(0.037)$ & \\
3) $\mathrm{BAY}-87-2243+\mathrm{RT} / \mathrm{BAY}-87-2243$ & $114.3[103 ; 132]$ & 1.07 \\
& $(0.4)$ & - \\
4) $\mathrm{RCT}$ & $99.5[83 ; 124]$ & \\
5) $\mathrm{RCT} / \mathrm{BAY}-87-2243$ & - & 0.93 \\
& $107.2[97 ; 123]$ & \\
6) BAY-87-2243+RCT/BAY-87-2243 & $(0.5)$ & $113.6[97 ; 145]$ \\
\hline
\end{tabular}

P-value $<0.05$ indicates significant differences between experimental treatment and respective control (RT or $\mathrm{RCT}$ ). we used fractionated irradiation which, in contrast to single dose irradiation used in our previous experiment [24], allows for specific scheduling evaluation and potential interaction with radiobiological mechanisms relevant to local tumor control after fractionated irradiation such as reoxygenation, repopulation, recovery and redistribution. The radiosensitizing effect was schedule-dependent and only significant if BAY-87-2243 was administered prior to fractionated irradiation. In contrast, concomitant administration of BAY-87-2243 during fractionated irradiation with or without cisplatin did not improve local tumor control. Whether pre-treatment with BAY87-2243 before start of radiochemotherapy affects local tumor control was not tested in the present experiments and remains to be elucidated. Thus, our data do not allow to assess whether the efficacy of BAY-87-2243 before initiation of fractionated radiotherapy depends on whether this is given alone or in combination with chemotherapy. When the experiments were designed we hypothesized based on our previous data [24] and findings by others that experimental arms 3 (BAY-87-2243+ RT/BAY-87-2243) and 6 (BAY-87-2243 + RCT/BAY-872243) are the most effective ones. However, unexpectedly concomitant BAY-87-2243 was not effective or even reversed the effect of pre-RT drug administration. Taken together, our data indicate a schedule dependence of the radiosensitizing effect of BAY-87-2243 but further experiments are necessary to confirm this conclusion.

The present data and our previous findings [24] suggest that the reduction in tumor hypoxia represents the underlying radiosensitizing mechanism of BAY-87-2243. Although we did not specifically investigate the mechanisms of reoxygenation induced by BAY-87-2243, the observed rapid drop in hypoxia already after 3 days of treatment with unchanged tumor vascular area and perfused functional vessels may suggest a cytotoxic effect of BAY-87-2243 on hypoxic tumor cells, e.g. blockage of HIF-1-mediated hypoxia tolerance [28]. However, necrotic fraction initially decreased and later remained unchanged in BAY-87-2243 treated tumors, it remains unclear to what extent rapid resorption of necrotic cells and/or reduction of proliferation and thereby oxygen consumption may have contributed to this observation. Normalisation of the microvasculature appears less likely to explain the reduction in hypoxia as longer treatments with BAY-87-2243 for 5 and 7 days resulted in a decrease of vascular area and unchanged perfused vessels. Reduction in tumor vascularization upon HIF-1 inhibition has been also demonstrated by others, which was associated with the decreased gene expression of angiogenic growth factors and decreased mobilization of circulating angiogenic cells [29]. In the latter study these effects on tumor vasculature contributed to the reduced growth of tumor xenografts similarly to our findings. 

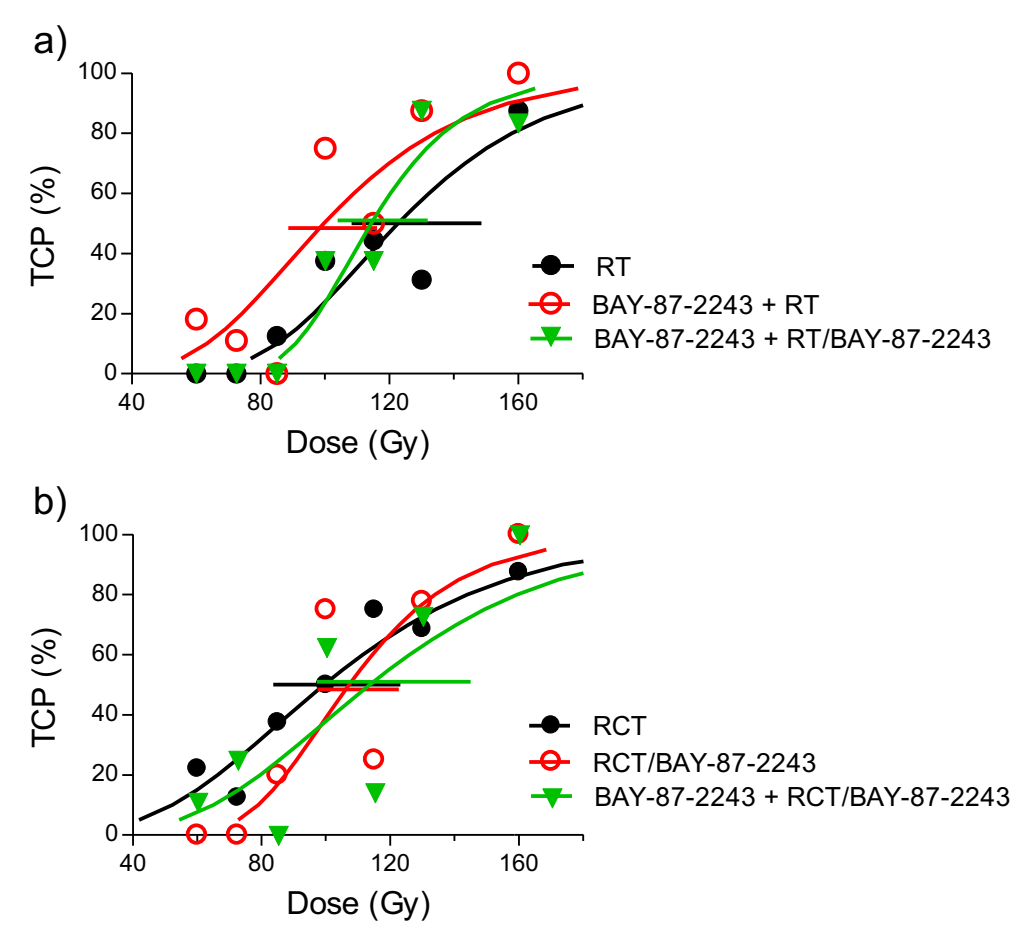

Figure 4 Effect of BAY-87-2243 on local tumor control. Dose-response curves after a) BAY-87-2243 combined with radiotherapy with 30 fractions within 6 weeks (RT) and b) BAY-87-2243 combined with fractionated radiochemotherapy with cisplatin (RCT). Symbols: observed local control rates; curves: calculated local tumor control probabilities (TCP); error bars: 95\% Cl of TCD50.

Another potential mechanism by which BAY-87-2243 reduces hypoxic fraction such as inhibition of mitochondrial oxygen consumption [23,30] appears rather less likely because UT-SCC-5 tumors exhibit very high glycolytic activity in vivo [31-33].

In our previous study pre-treatment with BAY-847296 for two weeks radiosensitized tumors to irradiation with large single doses resulting in an enhancement ratio of 1.37 [24], which indicates that the dose to achieve the same level of local tumor control could be reduced by a factor of 1.37 if tumors are pre-treated with the inhibitor. This significant radiosensitizing effect could be explained at least to some extent by the pronounced reduction of the fraction of radioresistant hypoxic tumor cells at time of irradiation. These previous findings were obtained using BAY-84-7296 and prompted us to evaluate here the effect of its derivative BAY-87-2243 on local tumor control after clinically relevant fractionated irradiation. BAY-87-2243 was chosen due to its higher ontarget activity for further experimental and clinical evaluation. The lead compound BAY-84-7296 was discovered by a small-molecule library screen for its activity to suppress HIF-1 $\alpha$ protein accumulation under hypoxic conditions. Subsequent studies on BAY-87-2243 revealed the mode of action, i.e. inhibition of mitochondrial complex I and reduced hypoxia-induced HIF-1 activity [23].
In the present study pre-treatment time with BAY-872243 was reduced to 3 days because we showed that shorter treatment is sufficient to resolve tumor hypoxia as detected by pimonidazole hypoxia marker. Pretreatment with BAY-87-2243 resulted in an enhancement ratio of 1.23 . This effect was less pronounced in comparison with ER of 1.37 obtained after large single radiation doses and BAY-84-7296. In addition to longer pre-treatment time with the compound in single dose experiment, several biological factors may contribute to this difference. First, radiation response after single doses may depend on the hypoxic fraction to a greater extent than the outcome of fractionated irradiation leading to a more pronounced radiosensitizing effect in a single dose setting. Extensive reoxygenation between radiation fractions in UT-SCC-5 [25] may at least in part explain lower ER. Second, it is well established that in addition to hypoxia other radiobiological mechanisms such as repopulation, repair, etc. determine tumor response to fractionated irradiation, which can attenuate the effect of the compound [34].

It has been shown that several other mechanisms can contribute to the enhanced tumour radiation response in combination with HIF-1 inhibition. This includes prevention of reconstitution of stromal function after radiation and further sensitization of tumour vessels to 
radiation damage upon HIF-1 inhibition $[10,17,18]$. These findings together with the data showing a radioprotective function of HIF- $1 \alpha$ in accumulation of DNA damage response proteins [35] led us to hypothesize that application of BAY-87-2243 during fractionated irradiation in addition to pre-treatment prior to radiotherapy further improves local tumor control. Despite of these potential mechanistic advantages, the latter treatment scheme however, was less efficacious than pre-treatment with BAY-87-2243 and resulted in an ER of only 1.07. The underlying reasons remain unclear. HIF- $1 \alpha$ expression in the present study markedly decreased during BAY-87-2243 treatment prior to irradiation and it was not tested here whether HIF- $1 \alpha$ recovered thereafter during irradiation to potentiate the effect of BAY-87-2243. However, we have previously shown that in UT-SCC-5 tumors despite of extensive reoxygenation during fractionated irradiation the levels of HIF-1 $\alpha$ remained unchanged [27] as opposed to HIF-1 up-regulation detected by others in other tumor cell lines $[6,8,16]$. Based on these findings, it may be hypothesised that limited expression of the molecular target during the course of radiotherapy underlies the lack of further improvement of local control. In addition it cannot be excluded that BAY-87-2243 during irradiation might induce adverse vascular effects with potentially negative consequences for perfusion and reoxygenation which may have attenuated the advantageous effect of pre-treatment with BAY-87-2243. Importantly, our finding that BAY-87-2243 is efficacious when given before but not during or after RT is in contrast to several other reports $[16,17,36]$. In the latter studies which used tumor growth delay as experimental endpoint, HIF-1 inhibition during and after RT but not prior to RT was efficacious. Whereas HIF-1 inhibition during radiotherapy may decrease the growth rate of surviving tumor cells influencing growth delay, it may not induce killing of tumor stem cells to an extent detectable as improved local tumor control. In addition one may speculate that the pharmacological effect of BAY-87-2243 on mitochondrial production of ROS and subsequent lower HIF-1 levels may have different radiobiological consequences than other HIF-1 targeting drugs or genetic knock-down.

Experimental evidence is accumulating that supports an important role of HIF-1 in chemoresistance by multiple mechanisms including increased drug efflux and/or decreased drug uptake, alteration of drug targets, cell cycle arrest in G1 affecting the cytotoxicity of cell phasespecific agents, etc. [37]. Deficiencies in nucleotide excision repair and in mismatch repair negatively affect the cytotoxicity of DNA interacting agents such as cisplatin [38]. HIF-1 has been shown in pre-clinical studies to interfere with these repair systems and it therefore has been suggested that HIF-1 inhibition can improve the outcome of radiochemotherapy [14,21,22,39]. In support of this hypothesis a recent clinical study has revealed a negative correlation between pre-treatment HIF- $1 \alpha$ expression in head and neck tumors and overall survival after concomitant cisplatin-based radiochemotherapy [40]. In line with other pre-clinical and clinical studies, our results show marginally significant enhancement of the radiation response by cisplatin [41-44]. BAY-87-2243 administered to tumor-bearing animals before and during fractionated cisplatin-based radiochemotherapy did not improve local tumor control in a single tumor hSCC. The mechanisms underlying the potential interactions between two different compounds and radiation and their net effect on local control have not been addressed in this study and need further investigations. Classical experiments have been reported that cisplatin showed greater enhancement in fully hypoxic (clamped) tumors than in tumors containing both hypoxic and well oxygenated cells (unclamped) [42]. It can be therefore speculated that in the present study pronounced reduction of hypoxic fraction by BAY-87-2243 might at least in part counteract the effect of cisplatin. It is however important to take into account considerable intertumoral heterogeneity in response to radiochemotherapy in the present study, which limits the interpretation of these data. Nevertheless, from a clinical point of view our results might have important implications for further development of this compound in the context of fractionated radiotherapy. While for radiochemotherapy it appears that caution and further experiments are needed, combination of compounds like BAY-87-2243 with radiation alone is of great clinical interest because many patients are medically unfit for concurrent radiochemotherapy. In addition, classical chemotherapy has substantial side effects and might exhibit only a limited benefit over radiotherapy alone with regard to local tumor control.

\section{Conclusion}

Pronounced reduction of tumor hypoxia by application of the novel inhibitor of hypoxia-induced gene activation BAY-87-2243 before start of fractionated radiotherapy improved local tumor control. The therapeutic effect of BAY-87-2243 importantly depended on treatment schedule and was only significant if BAY-87-2243 was administered prior to fractionated radiotherapy. The data support the further investigation of such inhibitors for combination with radiotherapy. In particular further radiobiological studies are needed to better understand the interaction of BAY-87-2243 with radiotherapy and radiochemotherapy which is a prerequisite for the development of predictive tests and tools for monitoring.

Competing interests

H. Hess-Stumpp and K. Unterschemmann are employees of Bayer Pharma AG. 


\section{Authors' contributions}

$\mathrm{LH}, \mathrm{DZ}, \mathrm{MB}, \mathrm{HHS}$ and $\mathrm{KU}$ conceived and designed the experiments; $\mathrm{LH}$ carried out most of the practical work and participated in data analysis. LK and KB participated in animal experiments. AY participated in histological study, performed data analysis; KG participated in the data analysis; LH, AY, DZ drafted the manuscript; HHS, KG, MB critically revised the manuscript. All authors read and approved the final manuscript.

\section{Authors' information}

AY and DZ - share senior authorship.

\section{Acknowledgements}

The excellent technical assistance of K. Schumann, E. Jung, D. Pfitzmann, S. Balschukat and L. Stolz-Kieslich is gratefully acknowledged. This study was supported in part by a research grant from Bayer Pharma AG and Sächsische Landesexzellenzinitiative (EU, Europäischer Fond für regionale Entwicklung "Europa fördert Sachsen!").

\section{Author details}

'OncoRay - National Center for Radiation Research in Oncology, Medical Faculty Carl Gustav Carus, TU Dresden, Dresden, Germany. ${ }^{2}$ Department of Radiation Oncology, University Hospital Carl Gustav Carus, TU Dresden, Dresden, Germany. ${ }^{3}$ German Cancer Consortium (DKTK), Dresden and German Cancer Research center (DKFZ), Heidelberg, Germany. ${ }^{4}$ Institute of Radiooncology Helmholtz-Zentrum Dresden-Rossendorf, Dresden, Germany. ${ }^{5}$ Global Drug Discovery, Bayer Pharma AG, Berlin and Wuppertal, Germany. ${ }^{6}$ Radiation Oncology, Eberhard Karls University Tübingen, Tübingen, Germany. ${ }^{7}$ German Cancer Consortium (DKTK), Tübingen, Germany. ${ }^{8}$ Department of Radiation Oncology (MAASTRO Lab), GROW - School for Oncology and Developmental Biology, Maastricht University Medical Centre, Maastricht, The Netherlands.

\section{Received: 1 July 2014 Accepted: 9 September 2014}

Published: 19 September 2014

\section{References}

1. Bachtiary B, Schindl M, Potter R, Dreier B, Knocke TH, Hainfellner JA, Horvat R, Birner P: Overexpression of hypoxia-inducible factor 1alpha indicates diminished response to radiotherapy and unfavorable prognosis in patients receiving radical radiotherapy for cervical cancer. Clin Cancer Res 2003, 9:2234-2240

2. Birner P, Schindl M, Obermair A, Breitenecker G, Oberhuber G: Expression of hypoxia-inducible factor 1alpha in epithelial ovarian tumors: its impact on prognosis and on response to chemotherapy. Clin Cancer Res 2001, 7:1661-1668.

3. Schindl M, Schoppmann SF, Samonigg H, Hausmaninger H, Kwasny W Gnant M, Jakesz R, Kubista E, Birner P, Oberhuber G: Overexpression of hypoxia-inducible factor 1alpha is associated with an unfavorable prognosis in lymph node-positive breast cancer. Clin Cancer Res 2002, 8:1831-1837.

4. Aebersold DM, Burri P, Beer KT, Laissue J, Djonov V, Greiner RH, Semenza GL: Expression of hypoxia-inducible factor-1alpha: a novel predictive and prognostic parameter in the radiotherapy of oropharyngeal cancer. Cancer Res 2001, 61:2911-2916.

5. dos Santos M, Mercante AM, Louro ID, Goncalves AJ, de Carvalho MB, da Silva EH, da Silva AM: HIF1-alpha expression predicts survival of patients with squamous cell carcinoma of the oral cavity. PLoS One 2012, 7:e45228.

6. Li F, Sonveaux P, Rabbani ZN, Liu S, Yan B, Huang Q, Vujaskovic Z, Dewhirst MW, Li CY: Regulation of HIF-1alpha stability through S-nitrosylation. Mol Cell 2007, 26:63-74.

7. Doe MR, Ascano JM, Kaur M, Cole MD: Myc posttranscriptionally induces HIF1 protein and target gene expression in normal and cancer cells. Cancer Res 2011, 72:949-957.

8. Moeller BJ, Cao Y, Li CY, Dewhirst MW: Radiation activates HIF-1 to regulate vascular radiosensitivity in tumors: role of reoxygenation, free radicals, and stress granules. Cancer Cell 2004, 5:429-441.

9. Majmundar AJ, Wong WJ, Simon MC: Hypoxia-inducible factors and the response to hypoxic stress. Mol Cell 2010, 40:294-309.

10. Schwartz DL, Powis G, Thitai-Kumar A, He Y, Bankson J, Williams R, Lemos R, Oh J, Volgin A, Soghomonyan S, Nishii R, Alauddin M, Mukhopadhay U,
Peng Z, Bornmann W, Gelovani J: The selective hypoxia inducible factor-1 inhibitor PX-478 provides in vivo radiosensitization through tumor stromal effects. Mol Cancer Ther 2009, 8:947-958.

11. Kessler J, Hahnel A, Wichmann H, Rot S, Kappler M, Bache M, Vordermark D: HIF-1alpha inhibition by siRNA or chetomin in human malignant glioma cells: effects on hypoxic radioresistance and monitoring via CA9 expression. BMC Cancer 2010, 10:605.

12. Staab A, Loeffler J, Said HM, Diehlmann D, Katzer A, Beyer M, Fleischer M, Schwab F, Baier K, Einsele H, Flentje M, Vordermark D: Effects of HIF-1 inhibition by chetomin on hypoxia-related transcription and radiosensitivity in HT 1080 human fibrosarcoma cells. BMC Cancer 2007, 7:213.

13. Dikmen ZG, Gellert GC, Dogan P, Yoon H, Lee YB, Ahn CH, Shay JW: In vivo and in vitro effects of a HIF-1alpha inhibitor, RX-0047. J Cell Biochem 2008, 104:985-994.

14. Sasabe E, Zhou X, Li D, Oku N, Yamamoto T, Osaki T: The involvement of hypoxia-inducible factor-1alpha in the susceptibility to gamma-rays and chemotherapeutic drugs of oral squamous cell carcinoma cells. Int I Cancer 2007, 120:268-277.

15. Song X, Liu X, Chi W, Liu Y, Wei L, Wang X, Yu J: Hypoxia-induced resistance to cisplatin and doxorubicin in non-small cell lung cancer is inhibited by silencing of HIF-1alpha gene. Cancer Chemother Pharmacol 2006, 58:776-784.

16. Harada H, Itasaka S, Zhu Y, Zeng L, Xie X, Morinibu A, Shinomiya K, Hiraoka M: Treatment regimen determines whether an HIF-1 inhibitor enhances or inhibits the effect of radiation therapy. Br J Cancer 2009, 100:747-757.

17. Moeller BJ, Dreher MR, Rabbani ZN, Schroeder T, Cao Y, Li CY, Dewhirst MW: Pleiotropic effects of HIF-1 blockade on tumor radiosensitivity. Cancer Cell 2005, 8:99-110.

18. Meijer TW, Kaanders JH, Span PN, Bussink J: Targeting hypoxia, HIF-1, and tumor glucose metabolism to improve radiotherapy efficacy. Clin Cancer Res 2012, 18:5585-5594.

19. Harada H, Inoue M, Itasaka S, Hirota K, Morinibu A, Shinomiya K, Zeng L, Ou G, Zhu Y, Yoshimura M, McKenna WG, Muschel RJ, Hiraoka M: Cancer cells that survive radiation therapy acquire HIF-1 activity and translocate towards tumour blood vessels. Nat Commun 2012, 3:783.

20. Tredan O, Galmarini CM, Patel K, Tannock IF: Drug resistance and the solid tumor microenvironment. J Natl Cancer Inst 2007, 99:1441-1454.

21. Su W, Huang L, Ao Q, Zhang Q, Tian X, Fang Y, Lu Y: Noscapine sensitizes chemoresistant ovarian cancer cells to cisplatin through inhibition of HIF-1alpha. Cancer Lett 2011, 305:94-99.

22. Liu Y, Bernauer AM, Yingling CM, Belinsky SA: HIF1alpha regulated expression of XPA contributes to cisplatin resistance in lung cancer. Carcinogenesis 2012, 33:1187-1192.

23. Ellinghaus $\mathrm{P}$, Heisler I, Unterschemmann $\mathrm{K}$, Haerter M, Beck H, Greschat S, Ehrmann A, Summer H, Flamme I, Oehme F, Thierauch K, Michels M, Hess-Stumpp H, Ziegelbauer K: BAY 87-2243, a highly potent and selective inhibitor of hypoxia-induced gene activation has antitumor activities by inhibition of mitochondrial complex I. Cancer Med 2013, 2:611-624.

24. Helbig L, Koi L, Bruchner K, Gurtner K, Hess-Stumpp H, Unterschemmann K, Pruschy M, Baumann M, Yaromina A, Zips D: Hypoxia-inducible factor pathway inhibition resolves tumor hypoxia and improves local tumor control after single-dose irradiation. Int J Radiat Oncol Biol Phys 2014, 88:159-166.

25. Yaromina A, Kroeber T, Meinzer A, Boeke S, Thames H, Baumann M, Zips D: Exploratory study of the prognostic value of microenvironmental parameters during fractionated irradiation in human squamous cell carcinoma xenografts. Int I Radiat Oncol Biol Phys 2011, 80:1205-1213.

26. Yaromina A, Zips D, Thames HD, Eicheler W, Krause M, Rosner A, Haase M, Petersen C, Raleigh JA, Quennet V, Walenta S, Mueller-Klieser W, Baumann M: Pimonidazole labelling and response to fractionated irradiation of five human squamous cell carcinoma ( $\mathrm{hSCC}$ ) lines in nude mice: the need for a multivariate approach in biomarker studies. Radiother Oncol 2006, 81:122-129.

27. Helbig L, Yaromina A, Sriramareddy SN, Boke S, Koi L, Thames HD, Baumann M, Zips D: Prognostic value of HIF-1alpha expression during fractionated irradiation. Strahlenther Onkol 2012, 188:1031-1037.

28. Wouters $B G$, Koritzinsky M: Hypoxia signalling through $m T O R$ and the unfolded protein response in cancer. Nat Rev Cancer 2008, 8:851-864.

29. Lee K, Qian DZ, Rey S, Wei H, Liu JO, Semenza GL: Anthracycline chemotherapy inhibits HIF-1 transcriptional activity and tumor-induced mobilization of circulating angiogenic cells. Proc Natl Acad Sci U S A 2009, 106:2353-2358 
30. Secomb TW, Hsu R, Ong ET, Gross JF, Dewhirst MW: Analysis of the effects of oxygen supply and demand on hypoxic fraction in tumors. Acta Oncol 1995, 34:313-316.

31. Sattler UG, Meyer SS, Quennet V, Hoerner C, Knoerzer H, Fabian C, Yaromina A, Zips D, Walenta S, Baumann M, Mueller-Klieser W: Glycolytic metabolism and tumour response to fractionated irradiation. Radiother Oncol 2010, 94:102-109.

32. Goetze K, Meyer SS, Yaromina A, Zips D, Baumann M, Mueller-Klieser W: Glycolysis-related gene induction and ATP reduction during fractionated irradiation: Markers for radiation responsiveness of human tumor xenografts. Strahlenther Onkol 2013, 189:782-788.

33. Yaromina A, Quennet V, Zips D, Meyer S, Shakirin G, Walenta S, MuellerKlieser W, Baumann M: Co-localisation of hypoxia and perfusion markers with parameters of glucose metabolism in human squamous cell carcinoma (hSCC) xenografts. Int J Radiat Biol 2009, 85:972-980.

34. Steel GG: Cell survival as a determinant of tumour response. In Basic clinical radiobiology. 3rd edition. Edited by Steel GG. London: Arnold; 2002:52-63.

35. Ayrapetov MK, Xu C, Sun Y, Zhu K, Parmar K, D'Andrea AD, Price BD: Activation of Hif1alpha by the prolylhydroxylase inhibitor dimethyoxalyglycine decreases radiosensitivity. PLoS One 2011, 6:e26064

36. Rouschop KM, Dubois $L$, Keulers TG, van den Beucken T, Lambin P, Bussink J, van der Kogel AJ, Koritzinsky M, Wouters BG: PERK/elF2alpha signaling protects therapy resistant hypoxic cells through induction of glutathione synthesis and protection against ROS. Proc Natl Acad Sci U S A 2013, 110:4622-4627.

37. Monti E, Gariboldi MB: HIF-1 as a target for cancer chemotherapy, chemosensitization and chemoprevention. Curr Mol Pharmacol 2011 4:62-77.

38. Martin LP, Hamilton TC, Schilder RJ: Platinum resistance: the role of DNA repair pathways. Clin Cancer Res 2008, 14:1291-1295.

39. Zhang N, Zhang H, Xia L, Zheng Y, Yu Y, Zhu Y, Chen G, Di W: NSC606985 induces apoptosis, exerts synergistic effects with cisplatin, and inhibits hypoxia-stabilized HIF-1alpha protein in human ovarian cancer cells. Cancer Lett 2009, 278:139-144.

40. van den Broek GB, Wildeman M, Rasch CR, Armstrong N, Schuuring E, Begg AC, Looijenga LH, Scheper R, van der Wal JE, Menkema L, van Diest PJ, Balm $A J$, van Velthuysen ML, van den Brekel MW: Molecular markers predict outcome in squamous cell carcinoma of the head and neck after concomitant cisplatin-based chemoradiation. Int J Cancer 2009, 124:2643-2650.

41. Bartelink H, Kallman RF, Rapacchietta D, Hart GA: Therapeutic enhancement in mice by clinically relevant dose and fractionation schedules of cis-diamminedichloroplatinum (II) and irradiation. Radiother Oncol 1986, 6:61-74.

42. Begg AC, Bohlken S, Bartelink H: The effect of cisplatin on the repair of radiation damage in RIF1 mouse tumours in vivo. Radiother Oncol 1989, 15:79-91.

43. Cooper JS, Zhang Q, Pajak TF, Forastiere AA, Jacobs J, Saxman SB, Kish JA, Kim HE, Cmelak AJ, Rotman M, Lustig R, Ensley JF, Thorstad W, Schultz CJ, Yom SS, Ang KK: Long-term Follow-up of the RTOG 9501/Intergroup Phase III Trial: Postoperative Concurrent Radiation Therapy and Chemotherapy in High-Risk Squamous Cell Carcinoma of the Head and Neck. Int J Radiat Oncol Biol Phys 2012, 84:1198-1205.

44. Pignon JP, le Maitre A, Maillard E, Bourhis J: Meta-analysis of chemotherapy in head and neck cancer (MACH-NC): an update on 93 randomised trials and 17,346 patients. Radiother Oncol 2009, 92:4-14.

doi:10.1186/1748-717X-9-207

Cite this article as: Helbig et al: BAY 87-2243, a novel inhibitor of hypoxia-induced gene activation, improves local tumor control after fractionated irradiation in a schedule-dependent manner in head and neck human xenografts. Radiation Oncology 2014 9:207.

\section{Submit your next manuscript to BioMed Central and take full advantage of:}

- Convenient online submission

- Thorough peer review

- No space constraints or color figure charges

- Immediate publication on acceptance

- Inclusion in PubMed, CAS, Scopus and Google Scholar

- Research which is freely available for redistribution

Submit your manuscript at www.biomedcentral.com/submit 